\title{
Study of Neutron From a Dense Plasma Focus Paco Instrument by Means of Nuclear Tracks Detectors
}

\section{MILANESE ${ }^{1}$, F. CASTILLO ${ }^{2 *}$, M. MOROSO ${ }^{1}$, M. BARBAGLIA ${ }^{1}$, J. I. GOLZARRI ${ }^{3,}$ H. MARTÍNEZ ${ }^{2}$, G. ESPINOSA ${ }^{3}$}

${ }^{1}$ Instituto de Física "Arroyo Seco" Universidad Nacional del Centro de la Provincia de Buenos Aires, Pinto 399, 7000 Tandil Argentina

${ }^{2}$ Instituto de Ciencias Físicas, Universidad Nacional Autónoma de México, Apartado Postal 48-3, 62251, Cuernavaca, Morelos, México

${ }^{3}$ Instituto de Física, Universidad Nacional Autónoma de México, Circuito de la Investigación Científica, Ciudad Universitaria, 04520, México D. F., México

*Email: ciro@fis.unam.mx

Published online: August 08, 2016,

The Author(s) 2016. This article is published with open access at www.chitkara.edu.in/publications

Abstract A most interesting feature of dense plasma foci is the acceleration of charge particle at energy in the range of $\mathrm{MeV}$ per nucleon. Using deuterium gas, this devices produce fusion D-D reactions, generation fast neutron pulses $(\sim 2.5 \mathrm{MeV})$. The device used in the present work is a Mather-type dense plasma focus, called PACO. It is a $2 \mathrm{~kJ}$ device at $31 \mathrm{kV}$, with an oxygen-free copper anode, $50 \mathrm{~mm}$ long with $40 \mathrm{~mm}$ diameter. The coaxial cathode is formed by ten copper rods arranged in a squirrel cage configuration at a radius of 50 $\mathrm{mm}$. The insulator in an annular Pyrex ${ }^{\circledR}$ tube located at the base of the anode. The energy store is provided by four $1 \mu \mathrm{F}(40 \mathrm{kV}, 40 \mathrm{nH})$ capacitors in parallel. The plasma focus was operated at $1.5 \mathrm{mb}$ deuterium gas pressure. Neutron and accelerated particles are analyzed with material detectors (CR-39 Lantrack $^{\circledR}$ ) for different conditions. A detailed study is made of track diameters when the plastic is chemically etched with, $6 \mathrm{~N} \mathrm{KOH}$ at $60^{\circ} \mathrm{C}( \pm 1)$ for $12 \mathrm{~h}$.

Keywords: Plasma focus; neutrons; CR-39; nuclear track detectors.

\section{INTRODUCTION}

The plasma focus was devised during the early days of fusion research [10,15], and immediately attracted the interest of researchers, due to the intense bursts of neutrons it emitted, from ${ }^{2} \mathrm{H}(\mathrm{d}, \mathrm{n})^{3} \mathrm{He}$ fusion reactions, when operated with deuterium gas. To date, it is arguably the most cost-effective plasma-based

Journal of Nuclear

Physics, Material Sciences, Radiation and Applications Vol-4, No-1, August 2016 pp. 89-98 
Milanese, $\mathrm{M}$. Castillo, F. Moroso, M. Barbaglia, M. Golzarri, J.I. Martínez, H. Espinosa, G.

neutron source, and although it is hardly a candidate for energy generation, it is attractive for other applications. A large number of experimental investigations have been conducted with the aim of better understanding the nature of the fusion mechanisms, and especially to test the validity of the thermonuclear model and various complementary models based on suprathermal mechanisms, such as the beam-target [2,18] and gyrating-particle models [13]. These studies have concentrated mainly on the neutron fluence characterization, including the neutron yield and anisotropy, the neutron energy spectra, and the temporal pulse duration and shape $[1,17,18]$. In particular, the neutron pulse duration ( $100 \mathrm{~ns})$ and the observed neutron anisotropy provide compelling evidence that supra-thermal mechanisms play an important role. However, in earlier work we found that there are both isotropic and anisotropic contributions in the neutron angular distribution, the former being the most important $[4,5]$. Although it has been established that there is more than one fusion mechanism, a detailed understanding of them is still lacking. In contrast to the neutron measurements, a paucity of experimental data exists on the charged fusion products: protons, tritons and ${ }^{3} \mathrm{He}$. As pointed out by Jäger et al. [14], such studies could provide much needed additional information on the fusion mechanisms. In the present work, in addition to the study of the neutron angular distribution, that of protons from the ${ }^{2} \mathrm{H}(\mathrm{d}, \mathrm{p})^{3} \mathrm{H}$ reaction channel is also studied, using a semi-circular holder. The fusion protons, with energies of $3.02 \mathrm{MeV}$, are far more penetrating than any of the other charged particles, except for the deuterium beam, which is axially accelerated by the focus. Therefore, they can be easily studied. The adverse environmental conditions encountered during a PF shot include: strong electromagnetic noise, intense emissions of light, ultra-violet, $\mathrm{x}$-rays and energeticelectrons. As mentioned above, there is also a strongaxially directed deuteron beam, which is followed by the expansion of hot plasma. The charged particle emission occurs in a time scale shorter than $10^{-7} \mathrm{~s}$, so the count-rate requirement for an electronic detector would be extremely high. In view of these considerations, it is apparent that polymer solid-state nuclear track detectors are well suited for time integrated diagnostics for neutrons and charged particles studied in plasma focus devices. Earlier work on the diagnostics of fusion neutrons, using CR-39 nuclear track detectors, was done by Collopy et al. [8], Frenje et al. [11], and Castillo etal. [4]. Charged particles incident on a polymer track detector, such as CR-39, deposit their kinetic energy as dense trails of ionization and excitation resulting in numerous polymer chain scissions. These damage trails represent latent particle tracks which can be made visible by chemical etching after the exposure [9]. Counting etched tracks by eye, using an optical microscope, has been the most frequently employed method 
for many years. Naturally, the tedious and laborious nature of such work is not convenient for the high accuracy required in the analysis of a large number of detectors. If the spatial distribution of the tracks is required, rather than simply a gross count, the task of manual analysis becomes extremely time-consuming and inconvenient. In the present work, an automated track measurement system has been employed, which overcomes the limitations of manual counting [12].

\section{EXPERIMENTAL SETUP}

The source of energetic neutrons used is a Mather type dense plasma focus device named PACO[15] with $2 \mathrm{~kJ}$ of energy stored in a capacitor bank and relatively small dimensions, placed in the National University of the Center of the Buenos Aires Province, Argentina. Anode (the usual): $\mathrm{OFHC} \mathrm{Cu}$, length: $50 \mathrm{~mm}$, diameter $40 \mathrm{~mm}$, hollow, with inner diameter of $20 \mathrm{~mm}$, covered by a disc Wf of $5 \mathrm{~mm}$ thick, inserted $5 \mathrm{~mm}$ deep from the end free anode and operated within the limits of deuterium pressure range with neutron production[16]. A schematic of the DPF PACO is shown in Figure 1.

This device has the feasibility of a simple mechanical modification of the support structure to allow its easy transportation. It normally operates as follows: a relatively fast discharge of high voltage $(31 \mathrm{kV})$ and current $(250 \mathrm{kA})$ is realized on a pair of coaxial electrodes under an atmosphere of

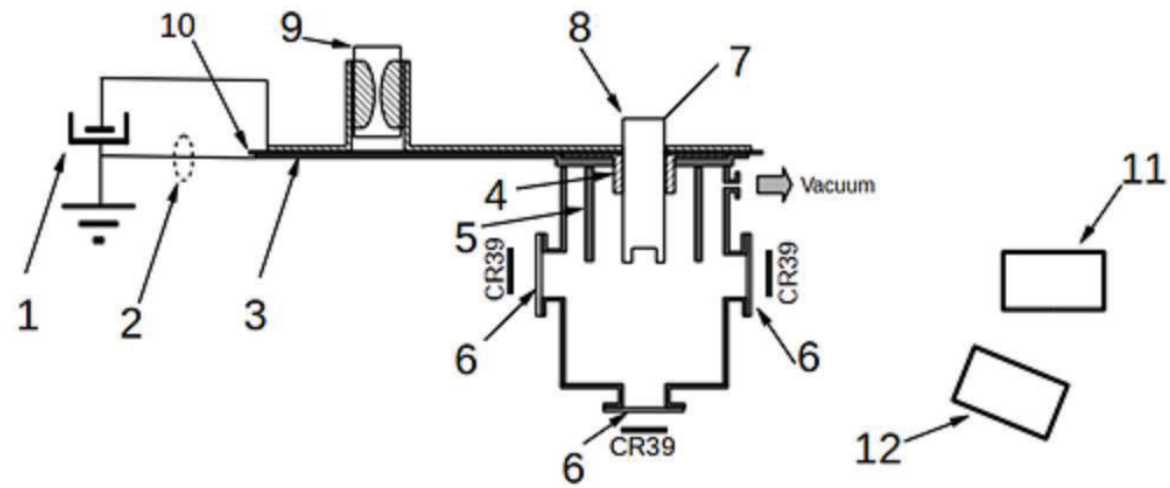

Figure 1: Schematic diagram of PACO plasma focus device. 1) capacitor bank, 2) Rogowski coil, 3) transmission line return path, 4) insulator, 5) cathode, 6) borosilicate window, 7) voltage probe, 8) anode, 9) spark-gap and 10) transmission line insulator (Mylar), 11) Fast Scintillator- Photomultiplier tube, 12) Silver Activation Counter neutron detector.
Study of Neutron

From a Dense

Plasma Focus Paco

Instrument by

Means of Nuclear

Tracks Detectors 
Milanese, $\mathrm{M}$. Castillo, F. Moroso, M. Barbaglia, M. Golzarri, J.I. Martínez, H. Espinosa, G.
$\mathrm{D}_{2}$ at low pressure (1-3 mbar). As a result of self-compression of the deuterium plasma, a zone of high density and temperature (focus) is formed where very energetic deuteron and electron beams, hard and soft X-ray pulses[7], and nuclear fusion reactions are generated. Therefore, a neutron flux of $2.45 \mathrm{MeV}$ is produced in pulsed form, with variable duration (shot to shot) between about 80 and $150 \mathrm{~ns}$ (FWHM) and a total average irradiance of about $2 \times$ $10^{8}$ neutrons per pulse distributed in $4 \pi \mathrm{sr}$.

Figure 2, shows typical waveforms, simultaneously recorded, of the temporal evolution of hard X-rays and fusion neutrons (lower trace), anodecathode voltage at the top of the coaxial gun (middle trace), and discharge current derivative (upper trace).

The diagnostics used for the registration of the current derivative and voltage are a calibrated Rogowski coil and a calibrated fast resistive voltage divider, respectively. The time integrated, in each pulse, neutron yield is registered by a calibrated Silver Activation Counter (SAC). The time resolved $\mathrm{X}$-ray pulses and neutrons are recorded by a plastic scintillator NE102A type,

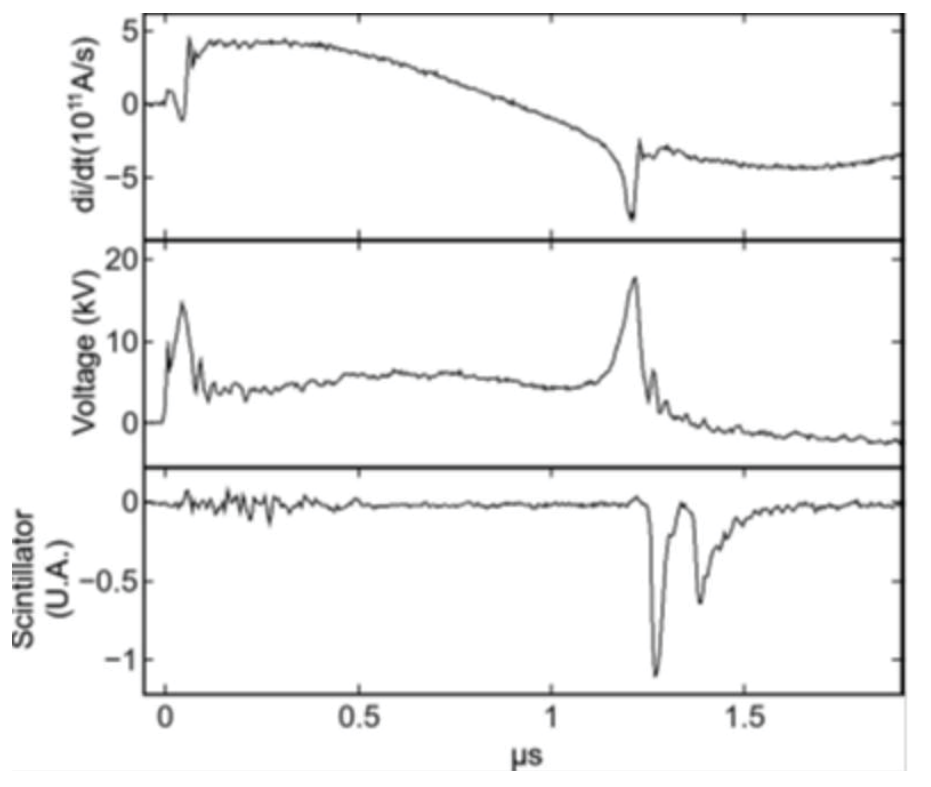

Figure 2: Waveforms corresponding to time resolved discharge current derivative (top), anode-cathode voltage taken at the top of the coaxial gun (middle), and hard X-rays and fusion neutrons (bottom). Temporal swept: 200ns/div. The hard X-ray and neutron pulses (lower trace) are separated by time of flight. 
optically coupled to a photomultiplier tube (S-PMT) located approximately $2 \mathrm{~m}$ from the emission source (focus). This distance can accurately separate both photon and neutron pulses by a difference of time-of-flight. X-ray emission is a secondary process that occurs almost simultaneously with neutron emission[3], as a result of the formation of a beam of very energetic (relativistic) electrons in the region of the dense and high temperature plasma during the final pinch stage. Experimental evidence suggests that its origin is associated with the Bremsstrahlung beam of electrons interacting with the back of the hollow central electrode (anode) in the PACO device. Due to the large flux of electrons and their high energy, an insert of a high atomic number material (tungsten) is placed inside to optimize the Bremsstrahlung conversion efficiency. The PACO device has a non-uniform angular distribution of the emission of neutrons. This was determined previously[6] using solid state nuclear track detectors CR-39.

The neutron flux $\phi$ averaged over several shots, was measured with CR-39 chips. This method has been used earlier at other devices to detect neutrons of energy around $2.45 \mathrm{MeV}$. CR-39 polycarbonate Lantrack $^{\circledR}$ chips, with a $1.9 \mathrm{~cm} \times 0.9 \mathrm{~cm}$ surface and $500 \mu \mathrm{m}$ thick as detection material, at two different angles relative to the gun axis: $0^{\circ}$, and $90^{\circ}$. Suitable neutron detection was obtained covering the CR-39 with $2 \mathrm{~mm}$ thick polyethylene sheets. The detectors were exposed to 150 shoots, for a filling pressure of $1.25 \mathrm{mbar}$. The chemical etching of the plastic chips was performed by immersing it in $\mathrm{KOH}$, $6.25 \mathrm{M}$ solution, at $60^{\circ} \mathrm{C} \pm 1{ }^{\circ} \mathrm{C}$ for 12 hours. The track counting was done with an optical microscope, with a 100X magnification, fixing a field of $2 \mathrm{~mm} \times$ $20 \mathrm{~mm}$ as measurement area. Forty fields per chip were counted, in order to obtain better statistics. The optical microscope is coupled to a CCD camera and a Digital Image Analysis System (DIAS), making all this measurements automatically. The software used for the counting tracks and track size analysis was Jandel Corporation Mocha ${ }^{\circledR}$ Image Analysis Software and Microsoft Excel $^{\circledR}$ for the data analysis.

\section{RESULTS}

Detectors CR-39 was placed at $0^{\circ}$ and $90^{\circ}$ with the system axis; outside the chamber discharges at a distance of $35 \mathrm{~cm}$ from the center electrode. It was determined that these angles were similarity detectors activated silver. The values obtained for the CR-39 in both cases are: at $0^{\circ}$ gives us an average 168 Traces $/ \mathrm{cm}^{2}$, and $90^{\circ}$ obtain 220 Traces $/ \mathrm{cm}^{2}$. This clearly defines us anisotropy.
Study of Neutron

From a Dense

Plasma Focus Paco Instrument by

Means of Nuclear

Tracks Detectors

\section{$\longrightarrow$}


Milanese, $\mathrm{M}$. Castillo, F. Moroso, M. Barbaglia, M. Golzarri, J.I. Martínez, H. Espinosa, G.
The average distribution of the neutron yield was studied using the CR-39 detectors. The distribution of the track per $\mathrm{cm}^{2}$ is shown in Figures 3 and 5, for each detector. The distribution of the track is shown in Figures 4 and 6, as position function. The uncertainties are greater difference between the average and the maximum and minimum values. When obtaining the track density by means of the Dias, only tracks whose ratio of minor diameter to major diameter was greater than 0.9 were considered.

A total of 21 detectors were exposed at $0^{\circ}$, representing an accumulated 188 ( $1.03 \times 10^{8}$ neutrons) PF shots. And a total of 28 detectors were exposed at $90^{\circ}$, representing an accumulated 180 (9.95 x 107 neutrons) PF shots. The CR39 detectors were etched in $6 \mathrm{M}-\mathrm{KOH}$ solutions at $\left(60 \pm 1^{\circ} \mathrm{C}\right)$ for $12 \mathrm{~h}$. A $6 \mathrm{M}$ solution of $\mathrm{KOH}$ is typically used as it has been shown that the sensitivity of CR-39, defined here as the ratio between the etch rate of a track and that of the bulk (VT/VB), is maximized at this molarity; higher sensitivity improves track contrast and the detection of charged particles with an optical microscope. The average number of counts N0 for the detector on axis, and N90 for the detector at $90^{\circ}$, were plotted as functions of the filling pressure for 390 shots. The data show a clearly defined anisotropy ratio $\mathrm{A}=\mathrm{N}_{0} / \mathrm{N}_{90}$, with a maximum around 1.5 mbar, which coincides with the pressure at which the maximum neutron yield is obtained.

The values obtained with CR-39 detectors were at $0^{\circ}$ to 222 tracks $/ \mathrm{cm}^{2}$ and $90^{\circ}$ was $168 \mathrm{tracks} / \mathrm{cm}^{2}$. What it gives us a neutron flux: $2.19 \times 10^{7} \mathrm{n}$ and 1.66

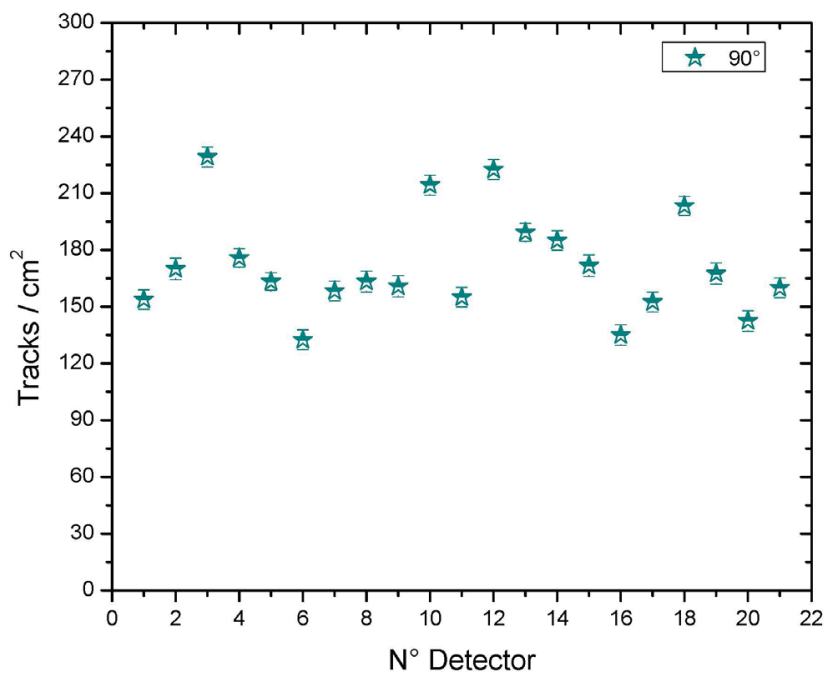

Figure 3: Tracks per $\mathrm{cm}^{2}$ for each detector at $90^{\circ}$. 


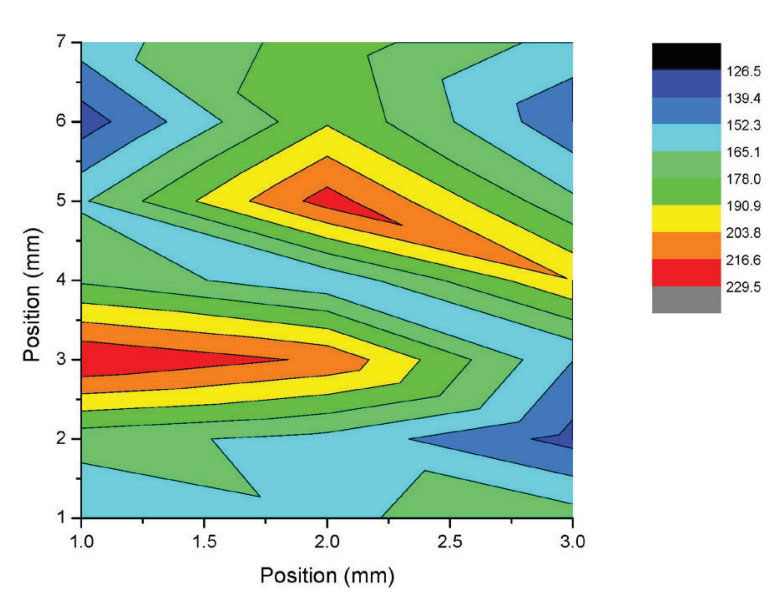

Study of Neutron

From a Dense

Plasma Focus Paco

Instrument by

Means of Nuclear

Tracks Detectors

Figure 4: Surface plot of the track numbers a function of $\mathrm{X}$ and $\mathrm{Y}$ position for each detectors at $90^{\circ}$.

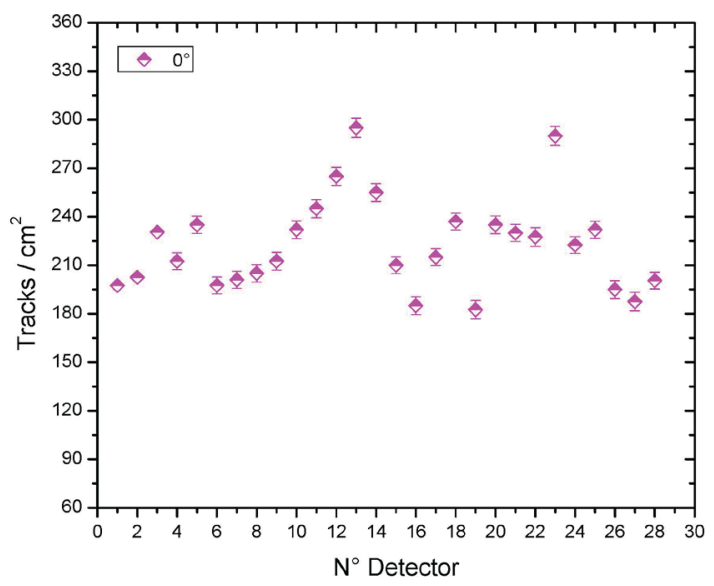

Figure 5: Tracks per $\mathrm{cm}^{2}$ for each detector at $0^{\circ}$.

$\mathrm{x} 10^{7} \mathrm{n}$ to zero and ninety degrees respectively. Which is consistent with those obtained by silver activated detectors which were at $0^{\circ}$ to 181 accounts, and $90^{\circ}$ of 165 accounts; giving a flow of $2.14 \times 10^{7} \mathrm{n}$ and $1.95 \times 10^{7} \mathrm{n}$ for each of the angles. 
Milanese, $\mathrm{M}$.

Castillo, F.

Moroso, M.

Barbaglia, M.

Golzarri, J.I.

Martínez, H.

Espinosa, G.

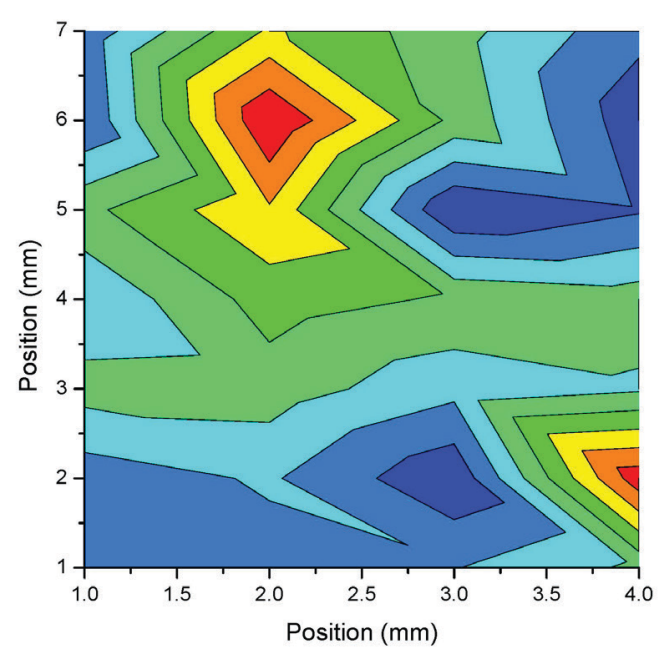

\begin{tabular}{|l|}
\hline 182.5 \\
\hline 196.6 \\
210.6 \\
224.7 \\
238.8 \\
252.8 \\
266.9 \\
280.9 \\
\hline 295.0 \\
\hline
\end{tabular}

Figure 6: Surface plot of the track numbers a function of $\mathrm{X}$ and $\mathrm{Y}$ position for each detectors at $0^{\circ}$.

The values obtained are in good agreement with previous results, and confirms that the team continues to perform well despite the changes. This time it was not possible to distinguish neutrons protons, possibly because the CR-39 detectors placed outside the chamber.

\section{CONCLUSIONS}

The responses obtained for CR-39 detectors exposed to neutron flux (2.45MeV) produced in the plasma focus PACO is quite consistent with the previously acquired. The small differences are due to position detector (CR-39) compared to the neutron flux. It is found in this work that the neutrons can account for at most one-tenth of the observed density. The nature of the array unfortunately forbids the possibility of exploring the yield at more angles. Most traces may be identified with accelerated protons along the axis. A smaller quality of traces cannot be identified with protons and may be due to accelerated impurities.

\section{ACKNOWLEDGMENTS}

The authors wish to thank Mr. Claudio Santiago, Mr. Lucas Conde, Mr. Marcelo Rodríguez, and Mr. Osvaldo Toscano for technical assistance and 
researchers of IFIMAT-UNICEN Institute for providing some material for measurement and to A. García, M.Veytia, P. Carrasco, N. Gonzalez, J. Martínez, and M. Cuautle, for their technical help. This work was partially supported by Dirección General del Personal Académico de la UNAM, Project IN103316. This work was supported in part by the Science and Technology Promotion National Agency of Argentina under Grant No. PICT 718-06.
Study of Neutron From a Dense Plasma Focus Paco Instrument by Means of Nuclear Tracks Detectors

\section{REFERENCES}

[1] Bernard, A., Coudeville, A., Jolas, A., Launspach, J. \& De Mascureau, J. Experimental studies of the plasma focus and evidence for non-thermal processes. Phys. Fluids 18,180 (1975). http://dx.doi.org/10.1063/1.861101

[2] Bernstein, M.J. \& Comisar, C.G. Neutron energy and flux distributions from a crossed-field acceleration model of plasma focus and z-pinch discharge.Phys. Fluids 15 700(1972). http://dx.doi.org/10.1063/1.1693966

[3] Bruzzone, H., et al. Temporal Correlations Between Hard X-Ray and Neutron Pulses in the PACO Plasma Focus Device. IEEE Trans. Plasma Sci. 38 (7), 1592 (2010). http://dx.doi.org/10.1109/TPS.2010.2049274

[4] Castillo, F., et al. Isotropic and anisotropic components of neutron emission at the FN-II and PACO dense plasma focus devices. Plasma Physics and Controlled Fusion 45289 (2003). http://dx.doi.org/10.1088/0741-3335/45/3/309

[5] Castillo, F., Milanese, M., Moroso, R., \&Pouzo, J. Evidences of thermal and nonthermal mechanisms coexisting in dense plasma focus D-D nuclear reactions. J. of Phys D: Appl. Phys. 33, 141 (2000). http://dx.doi.org/10.1088/0022-3727/33/2/308

[6] Castillo, F., Milanese, M., Moroso, R., \&Pouzo, J. Some experimental research on anisotropic effects in the neutron emission of dense plasma- focus devices. J. Phys. D: Appl. Phys. 30, 1499 (1997). http://dx.doi.org/10.1088/0022-3727/30/10/017

[7] Choi, P., Wong, C.S. \& Herold, H. Studies of the spatial and temporal evolution of a dense plasma focus in the x-ray region. Laser Part. Beams. 7, 763 (1989). http://dx.doi.org/10.1017/S0263034600006236

[8] Collopy, M.T, et al. Calibration of CR-39 for detecting fusion neutrons. Rev. Sci. Instrum. 63, 4892 (1992). http://dx.doi.org/10.1063/1.1143542

[9] Durrani, S.A. \& Bull, R.K. Solid State Nuclear Track Detection, (Pergamon Press, New York, 1987).

[10] Filippov, N.V., Fillippova, T.I.,\&Vinogradov, N.V.Dense high temperature plasma in a non-cylindrical z-pinch compression.Nuclear Fusion Suppl. 2, 577(1962).

[11] Frenjem, J.A.,et al., Absolute measurements of neutron yields from DD and DT implosions at the OMEGA laser facility using CR-39 track detectors. Rev. Sci. Instrum. 73, 2597 (2002). http://dx.doi.org/10.1063/1.1487889 
Milanese, $\mathrm{M}$. Castillo, F. Moroso, M. Barbaglia, M. Golzarri, J.I. Martínez, H. Espinosa, G.
[12] Gammage, R.B. \& Espinosa, G. Digital Imaging System for Track Measurements. Radiat.Meas. 28,835 (1997). http://dx.doi.org/10.1016/S1350-4487(97)00193-5

[13] Jäger, U. \& Herold, H. Fast ion kinetics and fusion reaction mechanism in the plasma focus. Nuclear Fusion.27, 407 (1987). http://dx.doi.org/10.1088/0029-5515/27/3/006

[14] Jäger, U., Bertalot, L. \& Herold, H. Energy spectra and space resolved measurements of fusion reaction protons from plasma focus devices. Rev. Sci. Instrum. 56, 77 (1985). http://dx.doi.org/10.1063/1.1138483

[15] Mather, J.W. Formation of a high-density deuterium plasma focus.Phys. Fluids, 8, 366 (1965). http://dx.doi.org/10.1063/1.1761231

[16] Pouzo, J., Cortázar, D., Milanese, M., Moroso, R. \&Píriz, A. Limits of deuterium pressure range with neutron production in plasma focus devices. Small Plasma Physics Experiments (World Scientific, London, 1988), p. 80.

[17] Steinmetz, K., Hubner, Rager, J.P. \&Robouch, B.V. Neutron pinhole camera investigation on temporal and spatial structures of plasma focus neutron source. Nuclear Fusion, 22, 25 (1982).

http://dx.doi.org/10.1088/0029-5515/22/1/003

[18] Tiseanu, I., Mandache, N \&Zambreanu V.Energetic and angular characteristics of the reacting deuterons in a plasma focus. Plasma Phys. Control Fission. 36,417(1994). http://dx.doi.org/10.1088/0741-3335/36/3/004 